International Journal of Pure and Applied Mathematics

Volume 103 No. 2 2015, 243-253

ISSN: 1311-8080 (printed version); ISSN: 1314-3395 (on-line version)

url: http://www.ijpam.eu

doi: http://dx.doi.org/10.12732/ijpam.v103i2.10

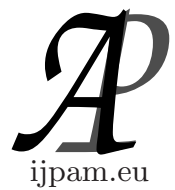

\title{
OSCILLATION OF FIRST ORDER NON-LINEAR NEUTRAL DIFFERENCE EQUATIONS OF RETARDED AND ADVANCED TYPE
}

\author{
A. Murugesan ${ }^{1}$, P. Selvakumar ${ }^{2}$ \\ ${ }^{1}$ Department of Mathematics \\ Government Arts College (Autonomous) \\ Salem, 636 007, Tamil Nadu, INDIA \\ ${ }^{2}$ Department of Mathematics \\ Dr. S.J.S. Paul Memorial College of \\ Engineering and Technology \\ Ramanathapuram Revenue Village \\ Villianur Commune, Puducherry, 605 502, Tamil Nadu, INDIA
}

\begin{abstract}
In this paper, we establish sufficient conditions for the oscillations for the all solutions of the first order non-linear neutral difference equations of retarded and advanced type. Our approach is to reduce the oscillation of neutral difference equations to the nonexistence of eventually positive solutions of non-neutral difference inequalities. Our results extend and improve several known results in the literature.
\end{abstract}

AMS Subject Classification: 39A10, 39A12

Key Words: first order difference equation, neutral, retarded, advanced, oscillation and nonoscillation properties

\section{Introduction}

We shall be concerned with the oscillatory behavior of solutions of the first

Received: April 13, 2015

(C) 2015 Academic Publications, Ltd.

${ }^{\S}$ Correspondence author url: www.acadpubl.eu 
order non-linear neutral difference equations of the retarded and advanced type

$$
\Delta[x(n)+p(n) x(n-\tau)]+\delta F\left(n, x^{\alpha}(n-\sigma)\right)=0,
$$

where $n \in N\left(n_{0}\right)=\left\{n_{0}+1, n_{0}+2, \ldots\right\}$ ( $n_{0}$ is a fixed nonnegative integer), $\delta= \pm 1, \alpha$ is a quotient of odd positive integers, $\Delta$ is the forward difference operator defined by $\Delta x(n)=x(n+1)-x(n)$, and the following conditions $\left(H_{1}\right)$ to $\left(H_{6}\right)$ are assumed to hold:

$\left(\mathrm{H}_{1}\right) \tau$ is a positive integer and $\sigma$ is an integer;

$\left(\mathrm{H}_{2}\right)\{p(n)\}$ is a sequence of real numbers;

$\left(\mathrm{H}_{3}\right)$ there exists a constant $p$ such that $-1<p \leq p(n) \leq 0$;

$\left(\mathrm{H}_{4}\right)$ for any $n \in N\left(n_{0}\right), F(n, x)$ is continuous in $x \in R$;

$\left(\mathrm{H}_{5}\right)$ there exists a sequence $\{q(n)\}$ of positive real numbers such that $|F(n, u)| \geq$ $q(n)|u|$;

$\left(\mathrm{H}_{6}\right) u F(n, u)>0$ for $n \in N\left(n_{0}\right)$ and $u \neq 0$.

If $\alpha=1$, then equation (1) becomes the equation.

$$
\Delta[x(n)+p(n) x(n-\tau)]+\delta F(n, x(n-\sigma))=0 .
$$

By a solution of equation (1) we mean a real sequence $\{x(n)\}$ which satisfies the equation (1) for which $\sup _{n \geq m}|x(n)|>0$ for any $m \in N\left(n_{0}\right)$. We assume that equation (1) always has such solutions. A solution of (1) is called non-oscillatory if it is eventually positive or eventually negative. Otherwise, it is called oscillatory. Equation (1) is called oscillatory if all its solutions are oscillatory.

In recent years there has been much interest in studying the oscillation of first order neutral delay and advanced difference equations. In particular Thandapani et al.[8] established sufficient conditions for oscillation of all solutions of equations (1) for the case $p(n)>0$ and

$$
F\left(n, x^{\alpha}(n-\sigma)\right)=q(n) x^{\alpha}(n-\sigma) .
$$

However very little is known about sufficient conditions for equation (1) to be oscillatory for the case $p(n)<0$ with $\sigma<0$. 
The purpose of this paper is to establish sufficient conditions for the oscillation of all solutions of equation (1) when $p(n)$ is negative, and $\sigma<0$ or $\sigma>0$. In Section 2, we reduce the oscillation of the neutral difference equation (1) to the nonexistence of eventually positive solution of non-neutral difference inequalities of the form

$$
\delta \Delta z(n)+q(n) z^{\alpha}(n-\beta) \leq 0
$$

where $\beta$ is an integer and $\{q(n)\}$ is a sequence of positive real numbers. Sufficient conditions for (3) to have no eventually positive solution have been established by many authors. For example see [1,3] and [5]. By combining these results with the results obtained in Section 2, we derive oscillation criteria for equation (1) in Section 3. The results obtained here extended and improve some of the results obtained in [8].

\section{Reduction to Non-Neutral Difference Inequalities}

In this section we consider the equation

$$
\Delta[x(n)+p(n) x(n-\tau)]+\delta F(n, f(x(n-\sigma)))=0
$$

where $\delta= \pm 1$, and conditions $\left(H_{1}\right)-\left(H_{6}\right)$ and the following conditions $\left(H_{7}\right)-$ $\left(H_{9}\right)$ are assumed to hold:

$\left(\mathrm{H}_{7}\right) f: R \rightarrow R$ is continuous, nondecreasing and $u f(u)>0$ for $u \neq 0$;

$\left(\mathrm{H}_{8}\right)$ there exists a continuous function $\varphi: R \rightarrow R$ such that $\varphi(u)$ is nondecreasing in $u \in R, u \varphi(u)>0$ for $u \neq 0$ and $|\varphi(u+v)| \leq|f(u)+f(v)|$ for $u v>0$;

$\left(\mathrm{H}_{9}\right)$ there exists a continuous function $w:(0, \infty) \rightarrow(0, \infty)$ such that $|f(u v)| \leq$ $w(u)|f(v)|$ for $u>0$ and $v \in R$.

Remark 1. For the case $f(u)=u^{\alpha}$, we can choose $\varphi(u)=\min \left\{1,2^{1-\alpha}\right\} u^{\alpha}$ and $w(u)=u^{\alpha}$.

Lemma 2. Suppose that $\delta=-1$ and $\left(H_{1}\right)-\left(H_{7}\right)$ hold. Let $\{x(n)\}$ be an eventually positive solution of (4) such that $\lim \sup _{n \rightarrow \infty} x(n)>0$. Set

$$
y(n)=x(n)+p(n) x(n-\tau) .
$$

Then $\{y(n)\}$ is eventually positive. 
Proof. Let $\{x(n)\}$ be an eventually positive solution of (1) such that $\limsup _{n \rightarrow \infty} x(n)>0$ and let $\{y(n)\}$ be its associated sequence defined by (5). Then the fact that $\Delta y(n)=F(n, f(x(n-\sigma)))>0$ for all large $n$, implies that $\{y(n)\}$ is of constant positive or constant negative sign eventually. This fact, in turn, implies that $\{y(n)\}$ is eventually positive or eventually negative.

If $y(n)>0$ does not hold then $y(n)<0$ eventually. If $\{x(n)\}$ is unbounded, then there exist a sequence $\left\{n_{k}\right\}$ of integers which tends to infinity and

$$
x\left(n_{k}\right)=\max _{n_{0} \leq n \leq n_{k}} x(n),
$$

such that $\lim _{k \rightarrow \infty} x\left(n_{k}\right)=+\infty$ Then, from (5), we have

$$
y\left(n_{k}\right)=x\left(n_{k}\right)+p\left(n_{k}\right) x\left(n_{k}-\tau\right) \geq x\left(n_{k}\right)(1+p) .
$$

From the above inequality, we obtain $\lim _{k \rightarrow \infty} y\left(n_{k}\right)=+\infty$ This is a contradiction. If $\{x(n)\}$ is bounded, then there is a sequence $\left\{n_{k}\right\}$ of integers which tends to infinity such that

$$
\lim _{k \rightarrow \infty} x\left(n_{k}\right)=\limsup _{n \rightarrow \infty} x(n)=L>0 .
$$

Since $\lim _{k \rightarrow \infty} x\left(n_{k}-\tau\right) \leq L$, then

$$
0 \geq \lim _{k \rightarrow \infty} y\left(n_{k}\right) \geq L(1+p)>0
$$

This is also a contradiction and the proof is complete.

Lemma 3. Suppose that $\delta=+1$, and $\left(H_{1}\right)-\left(H_{7}\right)$ hold. Let $\{x(n)\}$ be an eventually positive solution of (4) then $y(n)>0$ eventually.

Proof. Let $\{x(n)\}$ be an eventually positive solution of (4) and let $\{y(n)\}$ be its associated sequence defined by (5). Then, the fact that $\Delta y(n)=-F(n, f(x(n-$ $\sigma)))<0$, for all large $n$, which implies that $\{y(n)\}$ is strictly decreasing for all large $n$. Hence, if $y(n)>0$ does not hold, then $y(n)<0$ eventually and so there exist a constant $\mu>0$ such that $y(n) \leq-\mu$ for all large $n$, that is

$$
x(n) \leq-\mu-p(n) x(n-\tau),
$$

for all large $n$. We consider the following two possible cases.

Case 1: $\{x(n)\}$ is unbounded, i.e., $\lim \sup _{n \rightarrow \infty} x(n)=+\infty$. Thus, there exists 
a sequence $\left\{n_{k}\right\}_{k=1}^{\infty}$ of integers such that $n_{k} \rightarrow+\infty, x\left(n_{k}\right) \rightarrow+\infty$ as $k \rightarrow+\infty$ and $x\left(n_{k}\right)=\max _{n_{0} \leq n \leq n_{k}} x(n)$. Then, from (6), we have

$$
x\left(n_{k}\right) \leq-\mu-p\left(n_{k}\right) x\left(n_{k}-\tau\right)<-\mu+x\left(n_{k}\right),
$$

which is a contradiction.

Case 2: $\{x(n)\}$ is bounded, i.e. $\limsup _{n \rightarrow \infty} x(n)=l<\infty$. Choose a sequence of integers $\left\{\overline{n_{k}}\right\}_{k=1}^{\infty}$ such that $\overline{n_{k}} \rightarrow \infty$ and $x\left(\overline{n_{k}}\right) \rightarrow l$ as $k \rightarrow$ $\infty$. Let $m^{*}=\max \{\tau, \sigma\}, m=\min \{\tau, \sigma\}$ and $\xi_{k}$ be such that $x\left(\xi_{k}\right)=$ $\max \left\{x(n): \overline{n_{k}}-m^{*} \leq n \leq \overline{n_{k}}-m\right\}, \overline{n_{k}}-m^{*} \leq \xi_{k} \leq \overline{n_{k}}-m, k=1,2,3, \ldots$ Then $\xi_{k} \rightarrow \infty$ as $k \rightarrow \infty$ and $\lim \sup _{k \rightarrow \infty} x\left(\xi_{k}\right) \leq l$. Thus, by (6)

$$
x\left(\overline{n_{k}}\right) \leq-\mu-p\left(\overline{n_{k}}\right) x\left(\overline{n_{k}}-\tau\right) \leq-\mu+x\left(\xi_{k}\right) .
$$

Taking the superior limit as $k \rightarrow \infty$, we obtain

$$
l \leq-\mu+\limsup _{k \rightarrow \infty} x\left(\xi_{k}\right) \leq-\mu+l,
$$

which is also a contradiction. The proof is complete.

Theorem 4. Let $\delta=-1$. Suppose that $\left(H_{1}\right)-\left(H_{9}\right)$ hold. Then every solution of (4) is oscillatory or tends to zero if there exists a real sequence $\{\lambda(n)\}_{n=n_{0}}^{\infty}$ such that $0<\lambda(n)<1, n \in N\left(n_{0}\right)$ and the difference inequality

$$
\{-\Delta z(n)+Q(n) \varphi(z(n-\sigma))\} \operatorname{sgn} z(n) \leq 0
$$

does not have any nonoscillatory solution where

$$
Q(n)=\min \left\{\lambda(n) q(n), \frac{[1-\lambda(n-\tau)] q(n-\tau)}{w(p(n-\sigma))}\right\} .
$$

Proof. Assume the contrary. Without loss of generality, we may assume that the equation (4) has an eventually positive solution $\{x(n)\}$ which satisfies $\lim \sup _{n \rightarrow \infty} x(n)>0$. Then by Lemma $2, y(n)>0$ eventually and $\{y(n)\}$ is increasing for all large $n$, where $y(n)$ is defined by (5). Summing (4) from $N$ to $n-1$ yields

$$
\begin{aligned}
y(n) \geq & \sum_{s=N}^{n-1} F(s, f(x(s-\sigma))) \\
& \geq \sum_{s=N}^{n-1} q(s) f(x(s-\sigma))
\end{aligned}
$$




$$
\begin{aligned}
& =\sum_{s=N}^{n-1} \lambda(s) q(s) f(x(s-\sigma))+\sum_{s=N}^{n-1}[1-\lambda(s)] q(s) f(x(s-\sigma)) \\
= & \sum_{s=N}^{n-1} \lambda(s) q(s) f(x(s-\sigma))+\sum_{s=N+\tau}^{n-1}[1-\lambda(s-\tau)] q(s-\tau) f(x(s-\sigma-\tau)) \\
\geq & \sum_{s=N+\tau}^{n-1} Q(s) f(x(s-\sigma))+\sum_{s=N+\tau}^{n-1} Q(s) w(p(s-\sigma)) f(x(s-\sigma-\tau)) \\
\geq & \left.\sum_{s=N+\tau}^{n-1} Q(s) f(x(s-\sigma))+\sum_{s=N+\tau}^{n-1} Q(s) f(p(s-\sigma)) x(s-\sigma-\tau)\right) \\
\geq & \sum_{s=N+\tau}^{n-1} Q(s)[f(x(s-\sigma))+f(p(s-\sigma) x(s-\sigma-\tau))] \\
& \geq \sum_{s=N+\tau}^{n-1} Q(s) \varphi(x(s-\sigma)+p(s-\sigma) x(s-\sigma-\tau)) \\
& =\sum_{s=N+\tau}^{n-1} Q(s) \varphi(y(s-\sigma))
\end{aligned}
$$

for all large $n$. Put

$$
z(n)=\sum_{s=N+\tau}^{n-1} Q(s) \varphi(y(s-\sigma))>0 .
$$

Then $y(n) \geq z(n)$ and

$$
\Delta z(n)=Q(n) \varphi(y(n-\sigma) \geq Q(n) \varphi(z(n-\sigma))
$$

for all large $n$, so that $\{z(n)\}$ is an eventually positive solution of (7). This is a contradiction. The proof is complete.

Theorem 5. Let $\delta=+1$. Suppose that $\left(H_{1}\right)-\left(H_{9}\right)$ hold. Then every solution of (4) is oscillatory if there exists a real sequence $\{\lambda(n)\}_{n=n_{0}}^{\infty}$ such that $0<\lambda(n)<1, n \in N\left(n_{0}\right)$ and the difference inequality

$$
\{\Delta z(n)+Q(n) \varphi(z(n-\sigma+\tau))\} \operatorname{sgn} z(n) \leq 0
$$

has no nonoscillatory solution, where $Q(n)$ is defined by (8). 
Proof. Assume the contrary. Without loss of generality we may assume that $\{x(n)\}$ is an eventually positive solution of (4). Then by Lemma $2 y(n)>0$ eventually and $\{y(n)\}$ is increasing for all large $n$, where $y(n)$ is defined by (5). Summing (4) for $n$ to $\infty$, we have

$$
\begin{gathered}
y(n)=\lim _{n \rightarrow \infty} y(n)+\sum_{s=n}^{\infty} F(s, f(x(s-\sigma))) \\
\geq \sum_{s=n}^{\infty} q(s) f(x(s-\sigma))
\end{gathered}
$$

for all large $n$. By the same arguments as in the proof of Theorem 4, we find that

$$
y(n) \geq \sum_{n+\tau}^{\infty} Q(s) \varphi(y(s-\sigma)),
$$

for all large $n$. Put

$$
z(n) \geq \sum_{s=n}^{\infty} Q(s) \varphi(y(s-\sigma))>0 .
$$

Then $y(n) \geq z(n+\tau)$ eventually. We see that

$$
\Delta z(n)=-Q(n) \varphi(y(n-\sigma)) \leq-Q(n) \varphi(z(n-\sigma+\tau))
$$

for all large $n$, so that $\{z(n)\}$ is an eventually positive solution of (9). This is a contradiction. The proof is complete.

Applying Theorem 4 and 5 to equation (1), we have the following corollaries.

Corollary 6. Let $\delta=-1$. Suppose that $\left(H_{1}\right)-\left(H_{6}\right)$ hold. Then every solution of (1) is oscillatory or tends to zero if the difference inequality

$$
\Delta z(n)-P(n) z^{\alpha}(n-\sigma) \geq 0
$$

has no eventually positive solution where

$$
P(n)=\min \left\{1,2^{1-\alpha}\right\} \cdot \min \left\{\frac{q(n)}{1+[p(n-\sigma+\tau)]^{\alpha}}, \frac{q(n-\tau)}{1+[p(n-\sigma)]^{\alpha}}\right\}
$$

Corollary 7. Let $\delta=+1$. Suppose that $\left(H_{1}\right)-\left(H_{6}\right)$ hold. Then every solution of (1) is oscillatory if the difference inequality

$$
\Delta z(n)+P(n) z^{\alpha}(n-\sigma+\tau) \leq 0
$$

has no eventually positive solution, where $P(n)$ is defined by (10) 
Proof of Corollaries 6 and 7. We note that

$$
\delta \Delta z(n)+r(n) z^{\alpha}(n-\eta) \leq 0
$$

does not have any eventually positive solution if and only if

$$
\left\{\delta \Delta z(n)+r(n) z^{\alpha}(n-\eta) \leq 0\right\} \operatorname{sgn} z(n) \leq 0
$$

does not have any nonoscillatory solution, where $\eta$ is any integer and $\{r(n)\}$ is any real sequence. In fact, if $\{v(n)\}$ is an eventually negative solution of (12), then $z(n)=-v(n)$ is an eventually positive solution of (11). Therefore, the conclusions of Corollaries 6 and 7 following by applying Theorems 4 and 5 to equation (1) and by choosing

$$
\begin{aligned}
\varphi(u) & =\min \left\{1,2^{1-\alpha}\right\} u^{\alpha} \\
w(u)=u^{\alpha} \quad \text { and } \quad \lambda(n) & =\frac{1}{1+h^{\alpha}(n-\sigma+\tau)} \quad(\text { see Remark 2.1). }
\end{aligned}
$$

\section{Oscillation Theorems}

In this section we derive oscillation for equation (1). First we consider the case $\alpha \neq 1$. We need the following results which can be proved from the results that are proved in [5] for our subsequent discussion.

Lemma 8. Let $\alpha>1$ and $\sigma$ be a negative integer. Assume that $\left(H_{4}\right)-$ $\left(H_{6}\right)$ holds. Then the difference inequality.

$$
\Delta u(n)-F\left(n, u^{\alpha}(n-\sigma)\right) \geq 0 ; \quad n \in N\left(n_{0}\right)
$$

does not have any eventually positive solution if

$$
\sum_{n=n_{0}}^{\infty} q(n)=\infty
$$

Lemma 9. Let $0<\alpha<1$ and $\sigma$ be a positive integer. Assume that $\left(H_{4}\right)-\left(H_{6}\right)$ holds. Then the difference inequalilty.

$$
\Delta u(n)-F\left(n, u^{\alpha}(n-\sigma)\right) \leq 0 ; \quad n \in N\left(n_{0}\right)
$$

does not have any eventually positive solution if (13) holds. 
Combining Corollaries 6 and 7 with Lemmas 8 and 9, we derive the following theorems.

Theorem 10. Assume that $\left(H_{1}\right)-\left(H_{6}\right)$ holds. Suppose that $\delta=-1$, $\alpha>1$ and $\sigma$ is a negative integer. If

$$
\sum_{n=n_{0}}^{\infty} \min \left\{\frac{q(n)}{1+p^{\alpha}(n-\sigma+\tau)}+\frac{q(n-\tau)}{1+p^{\alpha}(n-\sigma)}\right\}=\infty,
$$

then every solution of equation (1) is oscillatory or tends to zero.

Theorem 11. Assume that $\left(H_{1}\right)-\left(H_{6}\right)$ holds. Suppose that $\delta=+1$, $0<\alpha<1$ and $\sigma>\tau$ if (14) hold, then every solution of equation (1) is oscillatory.

Corollary 12. Assume that $\left(H_{1}\right)-\left(H_{6}\right)$ holds. Suppose that $\delta=-1$, $\alpha>1, \sigma$ is a negative integer and

$$
\sum_{n=n_{0}}^{\infty} \min \{q(n), q(n-\sigma)\}=\infty .
$$

Then every solution of equation (1) is oscillatory or tends to zero.

Corollary 13. Assume that $\left(H_{1}\right)-\left(H_{6}\right)$ holds. Suppose that $\delta=+1$, $0<\alpha<1, \sigma$ is a positive integer, and (15) hold. Then every solution of equation (1) is oscillatory.

Proof of Corollaries 12 and 13. Since $\{p(n)\}$ is bounded for $n \in N\left(n_{0}\right)$, then it is easy to see that (15) implies (14). Hence, the conclusions of Corollaries 12 and 13 follows from Theorems 10 and 11 respectively.

Next we consider the equation (2). To prove our results we need the following lemmas which can be proved by the results that are proved in $[3,4]$.

Lemma 14. Assume that $\left(H_{4}\right)-\left(H_{6}\right)$ holds and $\sigma$ is a positive integer with $\sigma>1$. If

$$
\liminf _{n \rightarrow \infty} \sum_{s=n+1}^{n+\sigma-1} q(s)>\left(\frac{\sigma-1}{\sigma}\right)^{\sigma},
$$

then the difference inequality

$$
\Delta v(n)-F(n, v(n+\sigma)) \geq 0
$$

does not have any positive solution. 
Lemma 15. Assume that $\left(H_{4}\right)-\left(H_{6}\right)$ holds and $\sigma$ is a positive integer. If

$$
\liminf _{n \rightarrow \infty} \sum_{s=n-\sigma}^{n-1} q(s)>\left(\frac{\sigma}{\sigma+1}\right)^{\sigma+1}
$$

then the difference inequality

$$
\Delta u(n)-F(n, u(n-\sigma)) \leq 0
$$

does not have any positive solution.

Theorem 16. Let $\delta=-1$ and $\sigma<-1$. Assume that $\left(H_{1}\right)-\left(H_{6}\right)$ holds. If

$$
\lim _{n \rightarrow \infty} \sum_{s=n+1}^{n+\sigma-1} \min \left\{\frac{q(s)}{1+p(s-\sigma+\tau)}, \frac{q(s-\tau)}{1+p(s-\sigma)}\right\}>\left(\frac{\sigma-1}{\sigma}\right)^{\sigma}
$$

holds, then every solution of (2) is oscillatory or tends to zero.

Theorem 17. Let $\delta=+1, \sigma>0$ and $\sigma>\tau$. Assume that $\left(H_{1}\right)-\left(H_{6}\right)$ holds. If

$$
\lim _{n \rightarrow \infty} \sum_{s=n-\sigma+\tau}^{n-1} \min \left\{\frac{q(s)}{1+p(s-\sigma+\tau)}, \frac{q(s-\tau)}{1+p(s-\sigma)}\right\}>\left(\frac{\sigma}{\sigma+1}\right)^{\sigma+1}
$$

holds, then every solution of equation (2) is oscillatory.

Remark 18. Zhang [9] considered equation of type (1) and obtained oscillation results for the case

$$
F\left(n, x^{\alpha}(n-\sigma)\right)=q(n) x^{\alpha}(n-\sigma),
$$

$\{p(n)\}$ is a negative constant in $(0,1]$ and $\sigma$ is a positive integer whereas we obtain results for the case $\{p(n)\}$ is sequence of real numbers and $\sigma$ is a positive or negative integer.

\section{References}

[1] R. P. Agarwal, Difference Equations and Inequalities: Theory, Methods and Applications, Marcel Dekker, New York, 1999.

[2] Y. Gao and G. Zhang, Oscillation of nonlinear first order neutral difference equations, Appl. Math. E-Notes, 1(2001), 5-10. 
[3] I. Gyori and G. Ladas, Oscillation Theory of Delay Differential Equations with Applications, Clarendon Press, Oxford, 1991.

[4] Ch. G. Philos, On oscillation of some difference equations, Funkcial. Ekvac., 34(1991), 157-172.

[5] X. H. Tang and Y. Liu, Oscillation for nonlinear delay difference equation, Tamkang J. Math., 32 No. 4(2001), 275-280, doi:10.5556/j.tkjm.32.2001.342.

[6] X. H. Tang and Xiaoyan Lin, Necessary and sufficient conditions for oscillation of first - order nonlinear neutral difference equations, Comput. Math. Appl., 55(2008), 1279-1292, doi:10.1016/j.camwa.2007.06.009.

[7] X. H. Tang, J. S. Yu and D. H. Peng, Oscillation and nonoscillations of neutral difference equations with positive and negative coefficients, Comput. Math. Appl., 39(2000), 169-181.

[8] E. Thandapani, R. Arul and P. S. Raja, Oscillation of first order neutral delay differnce equations, Appl. Math. E-Notes, 3(2003), 88-94.

[9] G. Zhang, Oscillation of nonlinear neutral difference equations, Appl. Math. E-Notes, 2(2002), 22-24.

[10] Y. Zhou, Oscillation of neutral difference equations, Kyungpook Math. J., 39(1999), 283-291. 
